\title{
CARACTERÍSTICAS FÍSICAS DE FRUTOS E AMÊNDOAS E CARACTERÍSTICAS QUÍMICO-NUTRICIONAIS DE AMÊNDOAS DE ACESSOS DE SAPUCAIA ${ }^{1}$
}

\author{
VALDOMIROAURÉLIOBARBOSADE SOUZA², MICHELLE GARCÊZDECARVALHO, \\ KLEBER SILVASANTOS ${ }^{4}$, CLEMILTON DA SILVAFERREIRA $^{5}$
}

RESUMO - O objetivo deste trabalho foi avaliar a variabilidade fenotípica entre acessos de sapucaia para características físicas de frutos e amêndoas e características químico-nutricionais de amêndoas, para fins de uso em futuros trabalhos de melhoramento genético. Os frutos foram colhidos no estádio de pré-maturação e mantidos em temperatura ambiente por cerca de uma semana para completar a maturação. As seguintes características físicas e químico-nutricionais foram analisadas: peso médio de fruto, diâmetro longitudinal, diâmetro equatorial, relação diâmetro longitudinal/diâmetro equatorial do fruto, diâmetro da tampa, peso médio de amêndoa, comprimento da amêndoa, diâmetro da amêndoa, relação comprimento/diâmetro da amêndoa, número de amêndoas/fruto, energia, gordura, proteína bruta, cinzas e minerais. Os dados foram submetidos à análise de variância, e as médias dos acessos, comparadas pelo teste de Scott-Knott a 5\%. Houve diferenças estatísticas entre os acessos para as características avaliadas, exceto gordura e proteína bruta. Observou-se grande variabilidade fenotípica no germoplasma analisado, indicando que essa variabilidade pode servir de base inicial para futuros trabalhos de melhoramento genético.

Termos para indexação: Recursos genéticos, fruteira nativa, Lecythis pisonis, variabilidade fenotípica.

\section{FRUIT AND ALMOND PHYSICAL CHARACTERISTICS AND ALMOND CHEMICAL-NUTRITIONAL CHARACTERISTICS OF SAPUCAIA ACCESSES}

\begin{abstract}
The objective of this work was to evaluate fruit and almond physical characteristics and almond chemical-nutritional characteristics of sapucaia accesses. Fruits were harvested at the stage of pre-ripening and kept at environment temperature for about a week to complete the ripening. The following physical and chemical-nutritional characteristics were analyzed: fruit mean weight, fruit longitudinal diameter, fruit equatorial diameter, fruit longitudinal diameter/fruit equatorial diameter ratio, cover diameter, almond mean weight, almond length, almond diameter, almond length/diameter ratio, almond number/fruit; fat, brute protein, asher and minerals. The data was submitted to the variance analysis and access means compared by the Scott-Knott test at 5\%. There were statistical differences among accesses for all the analyzed characteristics, except for fat and brute protein. It was observed a great phenotypic variability in the analyzed germplasm, indicating that this variability may serve as a baseline for future works on genetic improvement.
\end{abstract}

Index terms: Genetic resources, native fruit tree, Lecythis pisonis, phenotypic variability.

\section{INTRODUÇÃO}

A variabilidade genética encontrada nas espécies frutíferas nativas é importante instrumento para enfrentar o aumento cada vez maior da demanda por alimentos. O Brasil, graças a sua localização geográfica e dimensão territorial, possui uma das maiores reservas de espécies nativas do mundo, com importantes centros de diversidade genética (Giacometti, 1993; Vieira, 1996).

A região amazônica é reconhecida como a principal reserva genética de plantas nativas do País, onde são encontradas mais de 500 espécies de frutas com potencial de uso pelo homem (Donadio, 1993; Giacometti, 1993). A segunda maior reserva genética de espécies nativas do Brasil está localizada nos cerrados (Giacometti, 1993; Assad, 1996; Ratter \& Ribeiro, 1996).

Existem, na flora nativa brasileira e, em especial, na Região
Meio-Norte do Brasil ou Nordeste Ocidental, constituída pelos Estados do Piauí e Maranhão, algumas espécies ainda pouco conhecidas, mas que apresentam potencialidades para o mercado de amêndoas. Dentre essas, podem ser mencionadas o chichá (Sterculia striata St. Hill. et Naud), a sapucaia (Lecythis pisonis Camb.) e a castanha-do-gurguéia (Dipteryx lacunifera Ducke) (Araújo, 1997), todas conhecidas e utilizadas pelas populações locais.

A sapucaia é uma espécie originária da Amazônia brasileira, ocorrendo desde o Ceará até o Rio de Janeiro, sendo particularmente freqüente no sul da Bahia e no norte do Espírito Santo. Ocorre, principalmente, em áreas de cerrado e de florestas pouco densas (Cavalcante, 1996). O fruto tem formato arredondado ou achatado, casca rígida e espessa e coloração castanha. Quando maduros, ocorre o fendilhamento em formato arredondado na parte inferior, semelhante a uma "tampa", por onde as sementes (amêndoas) são liberadas. As sementes são

${ }^{1}$ (Trabalho 041-08). Recebido em 18-02-2008. Aceito para publicação em: 05-08-2008.

${ }^{2}$ Eng. Agr., PhD., Embrapa Meio-Norte, Av. Duque de Caxias, 5650, CEP: 64006-220, Teresina-PI. E-mail: valdo@cpamn.embrapa.br

${ }^{3}$ Tecn. de Alimentos, Doutoranda em Ciência de Alimentos, FCF/USP, Bolsista CNPq, E-mail: michellegarcezpi@hotmail.com

${ }^{4}$ Graduando em Biologia, CCBS/UESPI, Estagiário da Embrapa Meio-Norte, Teresina-PI, E-mail: klebssantos@ hotmail.com

${ }^{5}$ Eng. Agr., Mestrando em Agronomia, CCA/UFPI, Bolsista da Capes E-mail: clemil@oi.com.br 
oleaginosas e apresentam coloração marrom-clara ou branca; podem ser consumidas cruas, cozidas ou torradas, constituindose em alimento saboroso e nutritivo (Teixeira, 2006). Contudo, ainda existem poucos dados científicos na literatura sobre os aspectos físicos do fruto e da amêndoa e do valor nutricional das amêndoas dessa espécie.

Portanto, é importante o desenvolvimento de pesquisas que visem a conhecer melhor essa espécie, principalmente nas áreas de recursos genéticos e aproveitamento agroindustrial. Essa necessidade adquire importância especial quando se considera o acelerado avanço nas taxas de ocupação do cerrado brasileiro (Machado et al., 2004).

Em geral, as amêndoas são ricas em proteínas e muito ricas em lipídios, não possuem colesterol, são boas fontes de fibras e possuem quantidades razoáveis de tiamina, riboflavina e niacina e de vitaminas B1 e B2. São ricas também em fósforo e potássio, mas pobres em sódio, favorecendo assim o bom funcionamento do sistema cardiovascular. Contêm, ainda, quantidades razoáveis de cálcio, magnésio e ferro (Benjamin, 1979). Algumas amêndoas, como é o caso da castanha-do-brasil (Souza \& Menezes, 2004) e da sapucaia (Vallilo et al., 1998), também são ricas em selênio, antioxidante cujo consumo vem sendo recomendado como forma de prevenção de câncer e doenças cardiovasculares.

Este trabalho teve como objetivo avaliar a variabilidade fenotípica existente entre acessos de sapucaia para características físicas de frutos e amêndoas e características químico-nutricionais de amêndoas, para fins de uso em futuros trabalhos de melhoramento genético.

\section{MATERIAL E MÉTODOS}

As análises das características físicas e químiconutricionais foram realizadas nos Laboratórios de Fisiologia Vegetal e Bromatologia da Embrapa Meio-Norte, em Teresina-PI, no período de junho de 2005 a março de 2006.

Foram analisados os frutos e as amêndoas de 23 acessos de sapucaia, coletados nos municípios de Altos $\left(05^{\circ} 02^{\prime} 17^{\prime \prime}\right.$ de latitude sul e $42^{\circ} 27^{\prime} 36^{\prime \prime}$ de longitude oeste), José de Freitas $\left(04^{\circ} 45^{\prime} 21^{\prime \prime}\right.$ de latitude sul e $42^{\circ} 34^{\prime} 33^{\prime \prime}$ de longitude oeste) e Teresina ( $05^{\circ} 05^{\prime} 13^{\prime \prime}$ de latitude sul e $42^{\circ} 48^{\prime} 41^{\prime \prime}$ de longitude oeste), no Estado do Piauí. Em 15 destes acessos (CPAMN 001, CPAMN 002, CPAMN 003, CPAMN 004, CPAMN 005, CPAMN 006, CPAMN 007, CPAMN 008, CPAMN 009, CPAMN 010, CPAMN 011, CPAMN 012, CPAMN 013, CPAMN 014, CPAMN 015), foram tomadas medidas de características físicas de frutos e amêndoas e, em 16 (CPAMN 003, CPAMN 004, CPAMN 005, CPAMN 006, CPAMN 008, CPAMN 012, CPAMN 013, CPAMN 015, CPAMN 016, CPAMN 017, CPAMN 018, CPAMN 019, CPAMN 020, CPAMN 021, CPAMN 022 e CPAMN 023), foram analisadas características químico-nutricionais.

Os frutos foram coletados no estádio de pré-maturação, transportados para o Laboratório de Fisiologia Vegetal da Embrapa Meio-Norte, onde foram mantidos em temperatura ambiente por cerca de uma semana, para completar a maturação.

Após a maturação dos frutos, executaram-se as seguintes medidas físicas: peso médio (PMF), diâmetro equatorial (DE), diâmetro longitudinal (DL), diâmetro da abertura (DAb), diâmetro da tampa (DTamp) e relação DL/DE. Posteriormente, as amêndoas foram extraídas manualmente e postas para secar em estufa com circulação forçada de ar a uma temperatura de $40^{\circ} \mathrm{C}$ por $24 \mathrm{~h}$. Completada a secagem, mediram-se: número de amêndoas/fruto (NA/F), peso total de amêndoas/fruto (PTA), relação PTA/PMF, peso médio de amêndoa (PMA), diâmetro da amêndoa (DA), comprimento da amêndoa (CA) e relação CA/DA. As medidas de PMF e PMA foram tomadas em balança digital com capacidade de $20 \mathrm{~kg}$ e balança digital semi-analítica com capacidade de 300 $\mathrm{g}$, respectivamente, e expressas em gramas. As demais medidas foram realizadas com o auxílio de um paquímetro digital e expressas em centímetros. Nessas avaliações, o tamanho da amostra analisada por acesso variou de um fruto (um acesso) a 11 frutos (12 acessos). Em três acessos, a amostra constituiu-se de oito frutos/acesso.

Após as análises físicas, parte das amêndoas de cada acesso foi utilizada na produção de mudas para formação de coleção de germoplasma, estabelecida em março de 2006, na área experimental da Embrapa Meio-Norte, em Teresina-PI. A outra parte das amêndoas foi embalada em recipientes plásticos e mantida em temperatura ambiente até o preparo para as análises químico-nutricionais.

O preparo das amostras para essa etapa do trabalho consistiu no descascamento das amêndoas e da trituração para a obtenção da pasta. Em seguida, as amostras foram identificadas e novamente acondicionadas em sacos plásticos herméticos e mantidas em freezer até o início dos procedimentos analíticos.

As seguintes características químico-nutricionais foram determinadas: proteína bruta (\%), gordura (\%), energia (kcal 100 $\mathrm{g}^{-1}$ ), cinzas (\%) e minerais ( $\mathrm{Ca}, \mathrm{Mg}, \mathrm{K}, \mathrm{P}, \mathrm{Mg}, \mathrm{Cu}$ e $\mathrm{Mn}$ ), expressos em mg $100 \mathrm{~g}^{-1}$. O teor de nitrogênio total foi determinado em $0,2 \mathrm{~g}$ da amostra pelo método Semimicro Kjeldahl (AOAC, 1992), utilizando-se de fator de multiplicação de 5,30 para transformação deste em proteína bruta. A determinação do teor de gordura (extrato etéreo ou lipídios) foi efetuada em extrator intermitente de Soxhlet, utilizando-se de éter de petróleo como solvente (Brasil, 2005). O teor de energia (valor calórico das amêndoas) foi calculado pela fórmula: $\mathrm{E}=[4$ (proteína + carboidrato $)+9$ (lipídios)], conforme Johannessen (1967) e Koziol \& Pedersen (1993); o teor de cinzas foi determinado pelo método de incineração adotado por Brasil (2005); o teor de minerais foi determinado de acordo com metodologia descrita em normas analíticas do Instituto Adolfo Lutz (Instituto Adolfo Lutz, 1985).

Os dados obtidos foram submetidos à análise de variância, utilizando-se do Programa SAS (SAS Institute, 1999). Nas determinações físicas, considerou-se um delineamento inteiramente ao acaso, com 15 tratamentos (acessos), onde frutos por acesso foram considerados como observações repetidas no mesmo indivíduo. Nas determinações químico-nutricionais, considerou-se, também, um delineamento inteiramente ao acaso, só que, neste caso, com 16 tratamentos e três repetições. As médias dos tratamentos (acessos) foram comparadas pelo teste de agrupamento Scott-Knott, a 5\%. 


\section{RESULTADOS E DISCUSSÃO}

Os resultados da análise de variância revelaram a existência de diferenças significativas entre acessos para todas as características físicas analisadas, exceto relação peso médio de fruto/peso total de amêndoas por fruto (relação PTA/PMF) (Tabelas 1, 2 e 3). Em termos de PMF, é possível classificar os acessos em quatro grupos: (1) frutos pequenos (PMF<1.100 g) acessos CPAMN 012, CPAMN 001, CPAMN 890,75, CPAMN 007 e CPAMN 015; (2) frutos médios (1.300 g $\leq \mathrm{PMF} \leq 1.500 \mathrm{~g})-$ acessos CPAMN 002, CPAMN 014, CPAMN 004 e CPAMN 008; (3) frutos grandes $(1.600 \mathrm{~g} \leq \mathrm{PMF} \leq 1.900 \mathrm{~g})-$ acessos CPAMN 013, CPAMN, 009 e CPAMN 006, e (4) frutos extragrandes (PMF $>2.000 \mathrm{~g}-$ acessos CPAMN 011, CPAMN 010 e CPAMN 005 (Tabela 1).

Os acessos CPAMN $006(29,35)$ e CPAMN $013(32,24)$ mostraram as maiores médias para número de amêndoas/fruto (NA/F), enquanto o acesso CPAMN $012(11,67)$ teve a menor média, sem, contudo, diferir estatisticamente de sete outros acessos. Por sua vez, o acesso CPAMN 013 (102,52 g) foi bem superior aos demais em termos de PTA, com média cerca de 57\% acima daquela obtida para o acesso CPAMN 006, o segundo de maior valor médio em termos absolutos para essa característica. Cerca de $67 \%$ dos acessos tiveram PTA inferior a $45 \mathrm{~g}$ e, conforme se pode observar pela Tabela 1, parece não haver correlação entre PTA e PMF e tendência de correlação negativa entre relação PTA/PMF e PMF. A baixa relação PTA/PMF indica que o rendimento médio de amêndoas da espécie ainda é pequeno. Por outro lado, a variação observada entre os acessos avaliados neste trabalho é um indicativo de que há espaço para ganhos em rendimento de amêndoas via melhoramento genético.

Os acessos CPAMN 006 (11,14 cm) e CPAMN 008 (11,45 $\mathrm{cm})$ tiveram as maiores médias de diâmetro da abertura (DAb), ao passo que o acesso CPAMN $006(12,83 \mathrm{~cm})$ apresentou a maior média para diâmetro da tampa (DTamp). Por sua vez, os acessos CPAMN $005(15,57 \mathrm{~cm})$ e CPAMN $014(15,58 \mathrm{~cm})$ sobressaíramse em relação aos demais em diâmetro longitudinal do fruto (DL), e os acessos CPAMN $010(19,42 \mathrm{~cm})$, CPAMN $005(18,52 \mathrm{~cm})$, CPAMN $011(18,34 \mathrm{~cm})$, CPAMN 009 (17,73 m) e CPAMN 006 $(16,75 \mathrm{~cm})$ sobressaíram-se em diâmetro equatorial do fruto (DE) (Tabela 2).

Quanto à relação DL/DE, os acessos CPAMN $013(1,07) \mathrm{e}$ CPAMN $014(1,09)$ apresentaram as maiores médias, enquanto os acessos CPAMN $010(0,69)$, CPAMN $011(0,69)$, CPAMN 009 $(0,68)$ e CPAMN $004(0,74)$ tiveram as menores médias para essa característica. A maioria dos acessos apresentou fruto de formato achatado, ou seja, com relação DL/DE inferior a 0,9. Apenas dois acessos (CPAMN 013 e CPAMN 014) mostraram frutos de formato arredondado (Tabela 2).

Na Tabela 3, estão apresentados os resultados das características físicas das amêndoas. O acesso CPAMN 013 está entre os que apresentaram as maiores médias para três das quatro características físicas avaliadas: peso médio de amêndoa (PMA) $(3,18 \mathrm{~g})$, comprimento da amêndoa (CA) $(2,85 \mathrm{~cm})$ e diâmetro da amêndoa (DA) $(1,48 \mathrm{~cm})$. Ainda em relação ao PMA, merecem destaque os acessos CPAMN 015 (2,78 g), CPAMN 011 (3,07 g) e CPAMN 014 (3,08 g). As menores médias para CA foram encontradas nos acessos CPAMN $004(1,91 \mathrm{~cm})$ e CPAMN 001 $(1,94 \mathrm{~cm})$. O acesso CPAMN $004(1,12 \mathrm{~cm})$ também apresentou a menor média de DA. Todos os acessos apresentaram amêndoas alongadas, ou seja, com relação CA/DA superior a 1. No entanto, os acessos CPAMN $005(2,21)$ e CPAMN $013(1,93)$ mostraram amêndoas mais alongadas que os demais.

Nas Tabelas 4 e 5, estão apresentados os resultados das características químico-nutricionais das amêndoas dos 16 acessos de sapucaia analisados. A quantidade de energia variou de 718,98 a 754,32 kcal $100 \mathrm{~g}^{-1}$, onde se sobressaíram os acessos CPAMN 008, CPAMN 013, CPAMN 017, CPAMN 018 e CPAMN 019 (Tabela 4). Esses resultados estão acima daqueles obtidos por Carvalho (2008) também para a sapucaia, mas com base numa mistura de amêndoas. Em comparação com outros tipos de amêndoas, pode-se considerar a sapucaia como excelente fonte de energia, com valores superiores aos citados por USDA (2007),

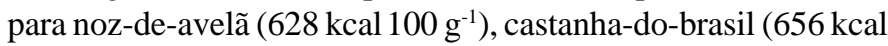

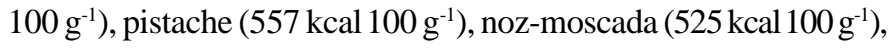
castanha-de-caju (574 kcal $\left.100 \mathrm{~g}^{-1}\right)$, amendoim (567 kcal $\left.100 \mathrm{~g}^{-1}\right)$ e noz-uropéia (612 kcal $\left.100 \mathrm{~g}^{-1}\right)$; por Philippi (2003), para castanha-

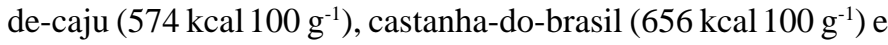
noz-pecã (667 kcal $\left.100 \mathrm{~g}^{-1}\right)$, e por Crepaldi et al. (2001), para

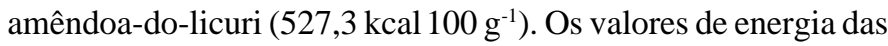
amêndoas de sapucaia também se equiparam aos mencionados por USDA (2007), para macadâmia (718 kcal $\left.100 \mathrm{~g}^{-1}\right)$ e noz-pecã (691 kcal $\left.100 \mathrm{~g}^{-1}\right)$, e por Philippi (2003) para macadâmia (702 kcal $\left.100 \mathrm{~g}^{-1}\right)$.

Não houve diferenças significativas entre acessos para os teores médios de gordura e proteína bruta (Tabela 4). Porém, o teor de gordura $(65,99 \%)$ obtido neste trabalho é superior ao obtido por Vallilo et al. (1998) para essa amêndoa (34,2-61,3\%) e, também, aos relatados na literatura para castanha-de-caju (43,047,7\%) (Sizaret \& Jardin, 1996; Melo et al., 1998; TACO, 2006; USDA, 2007), amendoim (49,66\%), noz-moscada $(36,31 \%)$, pistache $(44,44 \%)$, noz-européia $(56,96 \%)$ e avelã $(60,75 \%)$ (USDA, 2007); sendo equivalente àqueles relatados para castanha-dobrasil (63,5-66,43\%) (TACO, 2006; USDA, 2007), e inferior aos citados para noz-pecã $(71,97 \%)$ e macadâmia $(75,50-75,77 \%)$ (Philippi, 2003; USDA, 2007), indicando, portanto, que essa amêndoa é excelente fonte de gordura vegetal. Analisando mistura de amêndoas de sapucaia, Carvalho (2008) obteve 64,0\% de gordura, semelhante, portanto, ao obtido neste trabalho. Da mesma forma, o teor médio de proteína bruta $(19,62 \%)$ é o equivalente ao obtido por Carvalho (2008) e superior aos encontrados nas amêndoas do licuri (11,5\%) (Crepaldi et al., 2001); noz-moscada $(5,84 \%)$, macadâmia $(7,91 \%)$ e avelã $(14,95 \%)$ (USDA, 2007), e noz-pecã $(9,17-10,40 \%)$ e castanha-do-brasil $(14,32-17,00 \%)$ (Franco, 1992; USDA, 2007). No entanto, o teor protéico exibido na sapucaia é inferior aos encontrados por Lima et al. (2004), para a castanha-de-caju (24,5\%), e por USDA (2007) para o amendoim $(25,8 \%)$ e noz-européia $(24,9 \%)$.

No que se refere ao teor de cinzas, houve variação entre os acessos de 2,42\% a 4,44\%, onde o acesso CPAMN $018(2,42 \%)$ apresentou a menor média e diferiu estatisticamente dos demais (Tabela 4). O teor médio de cinzas $(3,43 \%)$ encontrado neste 
trabalho é equivalente àquele obtido por Carvalho (2008) com base numa mistura de amêndoas de sapucaia, e é superior aos encontrados por Lima et al. (2004) e Melo et al. (1998) para castanha-de-caju (2,50-2,52\%) e dos valores citados por USDA (2007) para macadâmia $(1,14 \%)$, noz-pecã $(1,49 \%)$, avelã $(2,29 \%)$ e noz-moscada $(2,34 \%)$, o que é um indicativo de que essa amêndoa é rica em minerais.

Os resultados obtidos nas análises de minerais estão apresentados na Tabela 5. As variações para os teores de cálcio e fósforo foram de 198,68 a 294,25 mg $100 \mathrm{~g} \mathrm{~g}^{-1}$ e de 557,47 a 998,26 $\mathrm{mg} 100 \mathrm{~g}^{-1}$, respectivamente. O acesso CPAMN 008 sobressaiuse em relação aos demais no teor de cálcio, enquanto os acessos CPAMN 016 e CPAMN 021 exibiram as maiores médias para o teor de fósforo. A maioria dos acessos apresentou teor de cálcio acima de $210 \mathrm{mg} 100 \mathrm{~g}^{-1}$, indicando que a amêndoa de sapucaia é bastante rica em cálcio, quando comparada com as principais amêndoas disponíveis no mercado, como macadâmia (40-85 mg $100 \mathrm{~g}^{-1}$ ) (Philippi, 2003; Morgano et al., 2007; USDA, 2007), avelã (144-155 mg $100 \mathrm{~g}^{-1}$ ), noz da nogueira (53-73 mg $100 \mathrm{~g}^{-1}$ ), pistache (103-115 mg $100 \mathrm{~g}^{-1}$ ) (Morgano et al., 2007; USDA, 2007), castanhado-brasil (160-172 mg $\left.100 \mathrm{~g}^{-1}\right)$ e noz-pecã (70-87 mg $\left.100 \mathrm{~g}^{-1}\right)$ (Franco, 1992; USDA, 2007), além da castanha-de-caju (146-165 mg $100 \mathrm{~g}^{-1}$ ) (Paiva et al., 2000; TACO, 2006). Em relação ao teor de fósforo, cerca de $50 \%$ dos acessos apresentaram teores médios desse mineral acima de $750 \mathrm{mg} 100 \mathrm{~g}^{-1}$, superando os valores de 520-620 mg $100 \mathrm{~g}^{-1}$ encontrados para essa espécie por Vallilo et al. (1998) e aqueles relatados por autores como Franco (1992), para castanha-do-brasil (746 mg $100 \mathrm{~g}^{-1}$ ) e noz-pecã (353 mg 100 $\mathrm{g}^{-1}$ ); Paiva et al. (2000), para castanha-de-caju (490 mg $100 \mathrm{~g}^{-1}$ ), e Morgano et al. (2007), para macadâmia (152-237 mg $100 \mathrm{~g}^{-1}$ ), avelã (376-418 mg $100 \mathrm{~g} \mathrm{~g}^{-1}$ ), pistache (424-530 mg $100 \mathrm{~g}^{-1}$ ) e noz-pecã (288-351 mg $\left.100 \mathrm{~g} \mathrm{~g}^{-1}\right)$. A maioria dos acessos também apresentou teores médios de fósforo superiores aos mencionados por USDA (2007) para as principais amêndoas.

Observa-se, também, ampla variação nos teores de potássio e magnésio, onde os acessos CPAMN $005(809,73 \mathrm{mg}$ $100 \mathrm{~g}^{-1}$ e 500,48 mg $\left.100 \mathrm{~g} \mathrm{~g}^{-1}\right)$, CPAMN 003 (738,42 mg $100 \mathrm{~g}^{-1} \mathrm{e}$

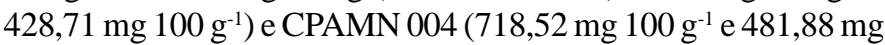
$\left.100 \mathrm{~g}^{-1}\right)$ sobressaíram-se nos teores desses dois minerais, e os acessos CPAMN 006 (753,15 mg $\left.100 \mathrm{~g}^{-1}\right)$, CPAMN $021(735,78 \mathrm{mg}$

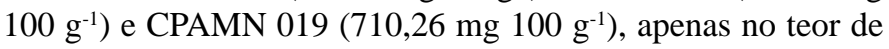

potássio. Os resultados obtidos neste estudo indicam que a amêndoa de sapucaia é bastante rica em potássio e magnésio (teores médios dos 16 acessos de 674,59 mg $100 \mathrm{~g}^{-1}$ e 449,89 mg $100 \mathrm{~g}^{-1}$, respectivamente) (Tabela 5), especialmente quando comparada com a noz-pecã (410-432 mg $100 \mathrm{~g}^{-1}$ e 121-125 mg 100 $\mathrm{g}^{-1}$ ) (Franco, 1992; USDA, 2007); a macadâmia (228-422 mg $100 \mathrm{~g}$ ${ }^{1}$ e 76-130 mg $100 \mathrm{~g} \mathrm{~g}^{-1}$ ) e a noz da nogueira (292-421 mg $100 \mathrm{~g}^{-1} \mathrm{e}$ 107-237 mg $100 \mathrm{~g}^{-1}$ ) (USDA, 2007). O maior teor de potássio, obtido pelo acesso CPAMN 005 (809,73 mg $\left.100 \mathrm{~g}^{-1}\right)$, é inferior àqueles relatados para a noz de pistache (910-1148 mg $\left.100 \mathrm{~g} \mathrm{~g}^{-1}\right)$, porém é superior aos teores médios citados para as demais amêndoas (Franco, 1992; Paiva et al., 2000; Philippi, 2003; USDA, 2007; Morgano et al., 2007).

Da mesma forma que para os demais minerais, a variação para os teores de cobre e manganês também foi significativa, indicando que há amplas possibilidades de sucesso no processo de seleção dentro do germoplasma estudado. $\mathrm{O}$ teor de cobre variou de 1,59 a 5,60 mg $100 \mathrm{~g}^{-1}$, enquanto o de manganês variou de 3,66 a 13,11 mg $100 \mathrm{~g}^{-1}$. O acesso CPAMN 004, além dos elevados teores de cobre e manganês, apresentou também teores elevados de potássio e magnésio. Os teores médios de cobre e manganês são bem superiores aos encontrados por Vallilo et al. (1998) e mais elevados também que os citados para macadâmia, avelã, noz-pecã, pistache, castanha-do-brasil, castanha-de-caju e noz-moscada (Morgano et al., 2007; USDA, 2007) em cerca de $87,5 \%$ e $62,5 \%$ dos acessos, respectivamente.

Relacionando-se os resultados das características físicas, em especial PMA, NA/F e PTA, com os das características químico-nutricionais, é possível evidenciar que os acessos CPAMN 013 e CPAMN 006 têm potencial de aproveitamento em futuros cultivos. O primeiro, apesar de não ter-se sobressaído em conteúdo de minerais, foi o que obteve maiores PMA, NA/F e PTA e o segundo, por seu valor nutricional. O acesso CPAMN 015, por sua vez, tem características nutricionais importantes e merece ser mais bem avaliado em termos de potencial agronômico. Finalmente, os acessos CPAMN 016, CPAMN 021, CPAMN 004 e CPAMN 012, em razão do elevado valor nutricional, têm importância para o melhoramento genético, a despeito da ausência de informações sobre as características físicas de amêndoas dos dois primeiros e do baixo desempenho dos dois últimos nessas características.

TABELA 1 - Peso médio de fruto (PMF), número de amêndoas/fruto, peso total de amêndoas (PTA) e relação PTA/PMF de 15 acessos de sapucaia. Teresina-PI, Embrapa Meio-Norte, 2007.

\begin{tabular}{|c|c|c|c|c|}
\hline $\operatorname{Acessos}^{1}$ & $\begin{array}{c}\text { PMF } \\
(\mathrm{g})\end{array}$ & NA/F & $\begin{array}{c}\text { PTA } \\
\text { (g) }\end{array}$ & $\begin{array}{c}\text { Relação } \\
\text { PTA/PMF }\end{array}$ \\
\hline CPAMN 005 & 2439,67 a & $13,67 \mathrm{c}$ & $29,12 \mathrm{c}$ & $0,01 \mathrm{a}$ \\
\hline CPAMN O10 & $2142,67 a$ & $16,67 \mathrm{c}$ & $31,51 \mathrm{c}$ & $0,01 \mathrm{a}$ \\
\hline CPAMN 011 & $2042,62 a$ & $18,35 \mathrm{c}$ & $56,33 \mathrm{~b}$ & $0,02 \mathrm{a}$ \\
\hline CPAMN 006 & $1894,44 \mathrm{~b}$ & $29,35 \mathrm{a}$ & $65,16 \mathrm{~b}$ & $0,04 \mathrm{a}$ \\
\hline CPAMN 009 & $1726,83 \mathrm{~b}$ & $24,20 \mathrm{~b}$ & $39,45 \mathrm{c}$ & $0,02 \mathrm{a}$ \\
\hline CPAMN 013 & $1648,85 b$ & $32,24 \mathrm{a}$ & $102,52 \mathrm{a}$ & 0,06 a \\
\hline CPAMN 008 & $1495,22 \mathrm{c}$ & $22,39 b$ & $43,21 \mathrm{c}$ & $0,04 \mathrm{a}$ \\
\hline CPAMN 004 & $1347,60 \mathrm{c}$ & $23,68 \mathrm{~b}$ & $37,18 \mathrm{c}$ & $0,04 a$ \\
\hline CPAMN 014 & $1343,65 c$ & $18,05 \mathrm{c}$ & $55,59 \mathrm{~b}$ & 0,04 a \\
\hline CPAMN 002 & $1302,27 \mathrm{c}$ & $15,67 \mathrm{c}$ & $33,38 \mathrm{c}$ & $0,02 \mathrm{a}$ \\
\hline CPAMN 015 & $1078,35 \mathrm{~d}$ & $21,39 \mathrm{~b}$ & $59,46 \mathrm{~b}$ & $0,05 \mathrm{a}$ \\
\hline CPAMN 007 & $907,05 \mathrm{~d}$ & $15,72 \mathrm{c}$ & $36,16 \mathrm{c}$ & $0,04 \mathrm{a}$ \\
\hline CPAMN 003 & $890,75 \mathrm{~d}$ & $12,93 \mathrm{c}$ & $27,67 \mathrm{c}$ & $0,03 \mathrm{a}$ \\
\hline CPAMN 001 & $787,97 \mathrm{~d}$ & $21,67 \mathrm{~b}$ & $38,14 \mathrm{c}$ & $0,04 \mathrm{a}$ \\
\hline CPAMN 012 & $649,97 \mathrm{~d}$ & $11,67 \mathrm{c}$ & $28,84 c$ & $0,04 a$ \\
\hline Média & 445,53 & 21,02 & 45,59 & $\mathrm{O}, 03$ \\
\hline C.V. $(\%)$ & 11,37 & 22,96 & 25,70 & 28,73 \\
\hline
\end{tabular}

${ }^{1}$ Médias seguidas da mesma letra, nas colunas, não diferem estatisticamente entre si, pelo teste de agrupamento Scott-Knott a $5 \%$. 
TABELA 2- Diâmetro da abertura (DAb), diâmetro longitudinal (DL), diâmetro equatorial (DE), diâmetro da tampa (DTamp) e relação DL/DE de frutos de 15 acessos de sapucaia. Teresina-PI, Embrapa Meio-Norte, 2007.

\begin{tabular}{crrrrr}
\hline Acessos $^{1}$ & $\begin{array}{c}\text { DAb } \\
(\mathrm{cm})\end{array}$ & $\begin{array}{c}\text { DL } \\
(\mathrm{cm})\end{array}$ & $\begin{array}{c}\text { DE } \\
(\mathrm{cm})\end{array}$ & $\begin{array}{c}\text { DTamp } \\
(\mathrm{cm})\end{array}$ & $\begin{array}{r}\text { Relação } \\
\text { DL/DE }\end{array}$ \\
\hline CPAMN 008 & $11,45 \mathrm{a}$ & $13,72 \mathrm{~b}$ & $14,51 \mathrm{~b}$ & $9,79 \mathrm{~b}$ & $0,95 \mathrm{~b}$ \\
CPAMN 006 & $11,14 \mathrm{a}$ & $14,27 \mathrm{~b}$ & $16,75 \mathrm{a}$ & $12,83 \mathrm{a}$ & $0,85 \mathrm{c}$ \\
CPAMN 014 & $9,24 \mathrm{~b}$ & $15,58 \mathrm{a}$ & $14,17 \mathrm{~b}$ & $10,08 \mathrm{~b}$ & $1,09 \mathrm{a}$ \\
CPAMN 013 & $9,15 \mathrm{~b}$ & $16,48 \mathrm{~b}$ & $15,24 \mathrm{~b}$ & $9,85 \mathrm{~b}$ & $1,07 \mathrm{a}$ \\
CPAMN 002 & $9,14 \mathrm{~b}$ & $11,97 \mathrm{c}$ & $14,62 \mathrm{~b}$ & $10,05 \mathrm{~b}$ & $0,82 \mathrm{c}$ \\
CPAMN 004 & $8,88 \mathrm{~b}$ & $11,24 \mathrm{~d}$ & $15,08 \mathrm{~b}$ & $7,87 \mathrm{~d}$ & $0,74 \mathrm{~d}$ \\
CPAMN 015 & $8,38 \mathrm{c}$ & $12,84 \mathrm{c}$ & $15,63 \mathrm{~b}$ & $9,09 \mathrm{c}$ & $0,82 \mathrm{c}$ \\
CPAMN 003 & $7,96 \mathrm{c}$ & $10,71 \mathrm{~d}$ & $12,83 \mathrm{~b}$ & $9,08 \mathrm{c}$ & $0,83 \mathrm{c}$ \\
CPAMN 007 & $7,73 \mathrm{c}$ & $11,85 \mathrm{c}$ & $13,64 \mathrm{~b}$ & $8,83 \mathrm{c}$ & $0,87 \mathrm{c}$ \\
CPAMN 005 & $7,69 \mathrm{c}$ & $15,57 \mathrm{a}$ & $18,52 \mathrm{a}$ & $9,33 \mathrm{c}$ & $0,84 \mathrm{c}$ \\
CPAMN 010 & $7,38 \mathrm{c}$ & $13,57 \mathrm{~b}$ & $19,42 \mathrm{a}$ & $9,21 \mathrm{c}$ & $0,69 \mathrm{~d}$ \\
CPAMN 011 & $7,33 \mathrm{c}$ & $12,69 \mathrm{c}$ & $18,34 \mathrm{a}$ & $9,04 \mathrm{c}$ & $0,69 \mathrm{~d}$ \\
CPAMN 009 & $6,83 \mathrm{c}$ & $12,07 \mathrm{c}$ & $17,73 \mathrm{a}$ & $8,85 \mathrm{c}$ & $0,68 \mathrm{~d}$ \\
CPAMN 012 & $6,69 \mathrm{c}$ & $9,87 \mathrm{~d}$ & $12,02 \mathrm{~b}$ & $8,41 \mathrm{~d}$ & $0,82 \mathrm{c}$ \\
CPAMN 001 & $5,09 \mathrm{~d}$ & $10,57 \mathrm{~d}$ & $11,85 \mathrm{~b}$ & $5,83 \mathrm{c}$ & $0,89 \mathrm{~b}$ \\
\hline Média & 6,27 & 12,85 & 15,36 & 9,21 & 0,84 \\
C.V. $(\%)$ & 5,71 & 5,79 & 6,12 & 6,24 & 2,77 \\
\hline
\end{tabular}

${ }^{1}$ Médias seguidas da mesma letra, nas colunas, não diferem estatisticamente entre si, pelo teste de agrupamento Scott-Knott a 5\%.

TABELA 3 - Características físicas de amêndoas de 15 acessos de sapucaia. Teresina-PI, Embrapa Meio-Norte, 2007.

\begin{tabular}{ccccc}
\hline Acessos $^{1}$ & $\begin{array}{c}\text { PMA } \\
(\mathrm{g})\end{array}$ & $\begin{array}{c}\text { CA } \\
(\mathrm{cm})\end{array}$ & $\begin{array}{c}\text { DA } \\
(\mathrm{cm})\end{array}$ & $\begin{array}{c}\text { Relação } \\
\text { CA/DA }\end{array}$ \\
\hline CPAMN 013 & $3,18 \mathrm{a}$ & $2,85 \mathrm{a}$ & $1,48 \mathrm{a}$ & $1,93 \mathrm{~b}$ \\
CPAMN 014 & $3,08 \mathrm{a}$ & $2,48 \mathrm{~b}$ & $1,45 \mathrm{a}$ & $1,71 \mathrm{c}$ \\
CPAMN 011 & $3,07 \mathrm{a}$ & $2,28 \mathrm{c}$ & $1,37 \mathrm{~b}$ & $1,67 \mathrm{c}$ \\
CPAMN 015 & $2,78 \mathrm{a}$ & $2,50 \mathrm{~b}$ & $1,55 \mathrm{a}$ & $1,61 \mathrm{~d}$ \\
CPAMN 012 & $2,48 \mathrm{~b}$ & $2,42 \mathrm{~b}$ & $1,39 \mathrm{~b}$ & $1,75 \mathrm{c}$ \\
CPAMN 007 & $2,30 \mathrm{~b}$ & $2,62 \mathrm{~b}$ & $1,47 \mathrm{a}$ & $1,78 \mathrm{c}$ \\
CPAMN 006 & $2,22 \mathrm{~b}$ & $2,53 \mathrm{~b}$ & $1,43 \mathrm{a}$ & $1,76 \mathrm{c}$ \\
CPAMN 003 & $2,14 \mathrm{~b}$ & $2,22 \mathrm{c}$ & $1,42 \mathrm{a}$ & $1,56 \mathrm{~d}$ \\
CPAMN 002 & $2,13 \mathrm{~b}$ & $2,11 \mathrm{~d}$ & $1,48 \mathrm{a}$ & $1,43 \mathrm{e}$ \\
CPAMN 005 & $2,13 \mathrm{~b}$ & $2,73 \mathrm{a}$ & $1,25 \mathrm{c}$ & $2,21 \mathrm{a}$ \\
CPAMN 008 & $1,93 \mathrm{c}$ & $2,25 \mathrm{c}$ & $1,46 \mathrm{a}$ & $1,53 \mathrm{~d}$ \\
CPAMN 010 & $1,89 \mathrm{c}$ & $2,17 \mathrm{c}$ & $1,40 \mathrm{~b}$ & $1,55 \mathrm{~d}$ \\
CPAMN 001 & $1,76 \mathrm{c}$ & $1,94 \mathrm{e}$ & $1,34 \mathrm{~b}$ & $1,44 \mathrm{e}$ \\
CPAMN 009 & $1,63 \mathrm{c}$ & $2,07 \mathrm{~d}$ & $1,34 \mathrm{a}$ & $1,55 \mathrm{~d}$ \\
CPAMN 004 & $1,57 \mathrm{c}$ & $1,91 \mathrm{e}$ & $1,12 \mathrm{~d}$ & $1,71 \mathrm{c}$ \\
\hline Média & 2,27 & 2,35 & 1,40 & 1,68 \\
C.V. $(\%)$ & 12,94 & 3,35 & 3,57 & 4,14 \\
\hline
\end{tabular}

${ }^{1}$ Médias seguidas da mesma letra, nas colunas, não diferem estatisticamente entre si, pelo teste de agrupamento Scott-Knott a 5\%. PMA = Peso médio de amêndoa; $\mathrm{CA}=$ Comprimento da amêndoa; DA = Diâmetro da amêndoa.

TABELA 4- Características químico-nutricionais de amêndoas de 16 acessos de sapucaia. Teresina-PI, Embrapa Meio-Norte, 2007.

\begin{tabular}{|c|c|c|c|c|}
\hline $\operatorname{Acessos}^{1}$ & $\begin{array}{c}\text { Energia } \\
\left(\mathrm{kcal} 100 \mathrm{~g}^{-1}\right)\end{array}$ & $\begin{array}{c}\text { Gordura } \\
(\%)\end{array}$ & $\begin{array}{c}\text { Proteína } \\
\text { bruta } \\
(\%)\end{array}$ & $\begin{array}{c}\text { Cinzas } \\
(\%)\end{array}$ \\
\hline CPAMN 013 & $754,32 \mathrm{a}$ & $65,14 a$ & 19,36 a & 3,42 a \\
\hline CPAMN 017 & 753,21 a & $68,34 \mathrm{a}$ & $19,72 \mathrm{a}$ & $2,87 a$ \\
\hline CPAMN O18 & $747,07 \mathrm{a}$ & 66,49 a & 19,92 a & $2,42 b$ \\
\hline CPAMN 019 & $746,66 \mathrm{a}$ & $66,13 \mathrm{a}$ & $20,50 \mathrm{a}$ & $2,97 a$ \\
\hline CPAMN 008 & $743,24 \mathrm{a}$ & $66,01 \mathrm{a}$ & $18,70 \mathrm{a}$ & 3,66 a \\
\hline CPAMN 015 & $740,50 \mathrm{~b}$ & $65,87 \mathrm{a}$ & $20,43 \mathrm{a}$ & 2,99 a \\
\hline CPAMN 022 & $737,07 \mathrm{~b}$ & $66,53 a$ & $19,97 \mathrm{a}$ & $3,82 \mathrm{a}$ \\
\hline CPAMN 020 & $736,39 \mathrm{~b}$ & $66,70 \mathrm{a}$ & $19,42 \mathrm{a}$ & 3,18 a \\
\hline CPAMN 003 & $734,98 \mathrm{~b}$ & 66,09 a & $19,47 \mathrm{a}$ & 3,40 a \\
\hline CPAMN 012 & $733,79 \mathrm{~b}$ & $67,51 \mathrm{a}$ & $21,51 \mathrm{a}$ & 3,12 a \\
\hline CPAMN 005 & $733,56 \mathrm{~b}$ & $65,86 a$ & $19,13 \mathrm{a}$ & $4,44 a$ \\
\hline CPAMN 006 & $732,96 \mathrm{~b}$ & $65,49 \mathrm{a}$ & 18,81 a & 3,93 a \\
\hline CPAMN 016 & $732,42 \mathrm{~b}$ & $65,60 \mathrm{a}$ & $20,23 \mathrm{a}$ & 3,42 a \\
\hline CPAMN 021 & $725,21 \mathrm{c}$ & $63,92 \mathrm{a}$ & $18,36 \mathrm{a}$ & 3,45 a \\
\hline CPAMN 004 & $719,63 \mathrm{c}$ & $65,49 \mathrm{a}$ & $19,47 \mathrm{a}$ & 4,05 a \\
\hline CPAMN 023 & $718,96 \mathrm{c}$ & $64,70 \mathrm{a}$ & $18,91 \mathrm{a}$ & $3,98 \mathrm{a}$ \\
\hline Média & 736,87 & 65,99 & 19,62 & 3,43 \\
\hline C.V. $(\%)$ & 3,35 & 4,59 & 8,77 & 11,16 \\
\hline
\end{tabular}

${ }^{1}$ Médias seguidas da mesma letra, nas colunas, não diferem estatisticamente entre si, pelo teste de agrupamento Scott-Knott a $5 \%$ 
TABELA 5- Teores de minerais em amêndoas de 16 acessos de sapucaia. Teresina-PI, Embrapa Meio-Norte, 2007.

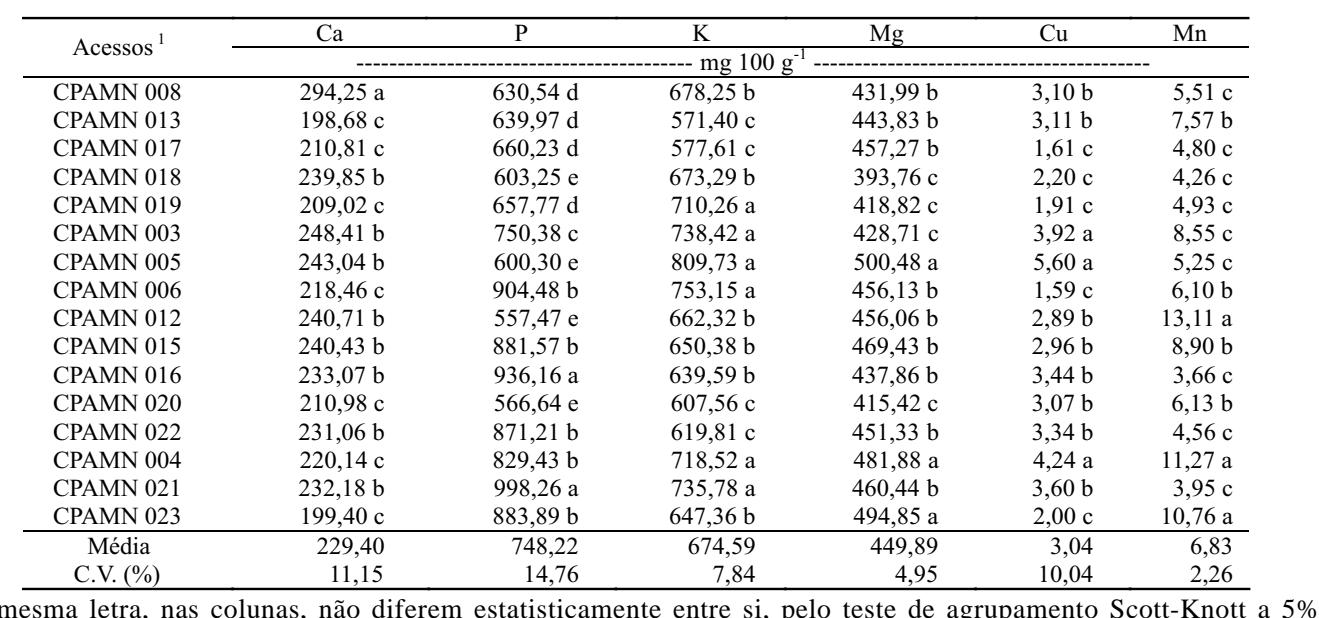

\section{CONCLUSÕES}

1-Os acessos de sapucaia apresentam variabilidade fenotípica significativa para a maioria das características analisadas, indicando que essa variabilidade pode servir de base inicial para futuros trabalhos de melhoramento genético.

2-Não houve relação direta de PMF com NA/F e PTA, ou seja, acessos com maiores médias de PMF não resultam, necessariamente, em maiores médias de NA/F e de PTA.

3-O acesso CPAMN 013, pela superioridade das características físicas de amêndoas como PMA, NA/F e PTA, e o acesso CPAMN 006, pelo valor nutricional, podem ser aproveitados em futuros cultivos de sapucaia.

\section{REFERÊNCIAS}

AOAC. Association of Official Analytical Chemists. Official methods of analysis. $12^{\text {th }}$ ed. Washington D.C, $1992.1115 \mathrm{p}$.

ARAÚJO, E.C.E. Chichá (Steculia striata St. Hil. et Naud.): uma nova opção para os mercados nacional e internacional de nozes. Informativo SBF, Caçador,. v.16, n.4, p.13-14, 1997.

ASSAD, M.L.R.C.L. Recursos biológicos: ocorrência e variabilidade. In: SIMPÓSIO SOBRE O CERRADO, 8., 1996, Brasília-DF. Anais ... Planaltina: EMBRAPA-CPAC, 1996. p.2024.

BENJAMIN, T.B. Nutrição humana. São Paulo, SP: McGraw-Hill do Brasil, 1979. 200p.

BRASIL. Ministério da Saúde. Agência Nacional de Vigilância Sanitária. Instituto Adolfo Lutz. Métodos físico-químicos para análise de alimentos. 4.ed. Brasília, 2005, p.105-119.

CARVALHO, M.G. Barras de cereais com amêndoas de chichá, sapucaia e castanha-do-gurguéia, complementadas com casca de abacaxi. 2008. 92 f. Dissertação (Mestrado) - Universidade
Federal do Ceará, Fortaleza, 2008. 92p.

CAVALCANTE, P.B. Frutas comestíveis da Amazônia. 6.ed. Belém: CNPq/Museu Paraense Emílio Goeldi, 1996. 282p.

CREPALDI, C.I.; MURADIAN, L.B.A.; RIOS, M.D.G.; PENTEADO, M.V.C.; SALATINO, A. Composição nutricional do fruto de licuri (Syagrus coronata (Martius) Beccari). Revista Brasileira de Botânica, São Paulo, v.24, n.2, p.155-159, 2001.

DONADIO, L.C. Frutíferas nativas da América Tropical. In: SIMPÓSIO NACIONAL DE RECURSOS GENÉTICOS DE FRUTEIRAS NATIVAS, 1993, Cruz das Almas-BA. Anais ... Cruz das Almas: EMBRAPA-CNPMF, 1993. p.9-12.

FRANCO, G. Nutriçãa: texto básico e tabelas de composição química dos alimentos. 9.ed. São Paulo, SP: Atheneu, 1992, 178p.

GIACOMETTI, D.C. Recursos genéticos de fruteiras nativas do Brasil. In: SIMPÓSIO NACIONAL DE RECURSOS GENÉTICOS DE FRUTEIRAS NATIVAS, 1993, Cruz das Almas, BA. Anais... Cruz das Almas: EMBRAPA-CNPMF, 1993. p.13-27.

INSTITUTO ADOLFO LUTZ. Normas analíticas do Instituto Adolfo Lutz: métodos químicos e físicos para análise de alimentos. 3.ed. São Paulo: Instituto Adolfo Lutz, 1985. 533p.

JOHANNESSEN, C.J. Pejibaye palm: physical and chemical analysis of the fruit. Economic Botany, Bronx, v.21, p.371-378, 1967.

KOZIOL, M.J.; PEDERSEN, H.B. Phytelephas aequatorialis Spruce (Arecaceae) in human and animal nutrition. Economic Botany, Bronx, v.47, p.401-407, 1993.

LIMA, A.C.; GARCIA, N.H.P.; LIMA, J.R. Obtenção e caracterização dos principais produtos do caju. Boletim CEPPA, Curitiba, v.22, n.1, p.133-144, 2004. 
MACHADO, R.B.; RAMOS NETO, M.B.; PEREIRA, P.G.P.; CALDAS, E.F.; GONÇALVES, D.A.; SANTOS, N.S.; TABOR, K.; STEININGER, M. Estimativas de perda da área do Cerrado brasileiro. Brasília: Conservação Internacional, 2004. 26p.

MELO, M.L.P.; MAIA, G.A.; SILVA, A.P.V.; OLIVEIRA, G.S.F.; FIGUEIREDO, R.W. Caracterização físico-química da amêndoa da castanha de caju (Anacardium occidentale L.) crua e tostada. Ciência e Tecnologia de Alimentos, Campinas, v.18, n.2, p.184187, 1998.

MORGANO, M.A.; SERAFIM, F.G.; FERREIRA, M.M.C.; PAULUCI, L.F.; SILVA, M.G.; MANTOVANI, D.M.B. Caracterização mineral de diferentes nozes. Disponível em: $\langle$ http://pcserver.iqm.unicamp.br/ marcia/Puban11.pdf $>$. Acesso em: 08 out. 2007.

PAIVA, F.F.A.; GARRUTTI, D.S.; SILVA NETO, R.M. Aproveitamento industrial do caju. Fortaleza: Embrapa Agroindústria Tropical, 2000. 88p.

PHILIPPI, S.T. Nutrição e técnica dietética. Barueri, SP: Monole, 2003.373p.

RATTER, J.A.; RIBEIRO, J.F. Biodiversity of the flora of the cerrado. In SIMPÓSIO SOBRE O CERRADO, 8., 1996, Brasília, DF. Anais ... Planaltina: EMBRAPA-CPAC, 1996. p.3-5.

SAS Institute. SAS/STAT user's guide 8,0. Cary, NC: SAS Institute, 1999. 3365p.
SIZARET, F; JARDIN, C. Estudo nacional da defesa familiar: tabelas de composição de alimentos. 4.ed. Rio de Janeiro: IBGE, 1996. 195p.

SOUZA, M.L.; MENEZES, H.C. Processamentos de amêndoas e torta de castanha-do-brasil e farinha de mandioca: parâmetros de qualidade. Ciência e Tecnologia de Alimentos, Campinas, v.4, n.1,p.120-128, 2004.

TACO. Tabela brasileira de composição de alimentos. 2.ed. v.2. Campinas: NEPA-UNICAMP, 2006. 113p.

TEIXEIRA, E. Frutas do Brasil: sapucaia. Disponível em:<http:/ /www.bibvirt.futuro.usp.br/ especiais/frutasnobrasil/ sapucaia.htm>. Acesso em: 16 out. 2006.

USDA. United States Department of Agriculture, Agricultural Research Service National Nutrient database for standard teference, Release 20, 2007. Disponível em: <http:// www.ars.usda.gov/ba/bhnrc/ndl>. Acesso em: 20 jan. 2008.

VALLILO, M.I.; TAVARES, M; AUED-PIMENTEL, S.; CAMPOS, N.C.; MOITA NETO, J.M. Lecythis pisonis Camb. nuts: oil characterization, fatty acids and minerals. Food Chemistry, Kidlington, v.66, n.2, p.197-200, 1998.

VIEIRA, R.F. Aspectos práticos da coleta de germoplasma. In: PUIGNAN, J.P.; CUNHA, R. da (Ed.). Conservacion de germoplasma vegetal. Montevideo: IICA/PROCISUR, 1996. p.7584. (Dialogo, 45). 